\title{
Impact de la castration du daguet rusa sur les performances de croissance, d'abattage et sur la qualité biologique de la viande à 24 et 30 mois
}

\author{
S. Le Bel ${ }^{1}$, P. Chardonnet ${ }^{2 *}$, D. Dulieu ${ }^{1}$, M. Salas ${ }^{1}$
}

LE BEL (S.), CHARDONNET (P.), DULIEU (D.), SALAS (M.). Impact de la castration du daguet rusa sur les performances de croissance, d'abattage et sur la qualité biologique de la viande à 24 et 30 mois. Revue Élev. Méd. vét. Pays trop., 1995, 48 (1) : 85-93

Chez le jeune mâle rusa (Cervus timorensis russa) destiné à être abattu, le rut est considéré par l'éleveur comme une contrainte. Le but de l'expérimentation est d'apprécier l'impact́ zootechnique de la castration. Dix-sept cerfs rusa de Java castrés (C) et dix-neuf entiers ( $E$ ), âgés de 12 mois ont été embouchés à l'herbe et abattus à 24 ou 30 mois. La castration induit une diminution du poids vif à 24 mois $(\mathrm{C}$ : $54,5 \pm 1,9 \mathrm{~kg}, \mathrm{E}: 58,5 \pm 2,3 \mathrm{~kg}, \mathrm{p}<0,01)$ et 30 mois $(\mathrm{C}: 62,5 \pm 3,0$ $\mathrm{kg}, \mathrm{E}: 68,5 \pm 2,3 \mathrm{~kg}, \mathrm{p}<0,01)$. De 12 à 24 mois, la croissance est de $82 \pm 3 \mathrm{~g} / \mathrm{j}(\mathrm{C})$ et $91 \pm 1 \mathrm{~g} / \mathrm{j}(\mathrm{E})$. De 24 à 30 mois, elle ralentit pour n'atteindre plus que $45 \pm 6 \mathrm{~g} / \mathrm{j}$ (C) et $63 \pm 12 \mathrm{~g} / \mathrm{j}(\mathrm{E})$. La castration et le vieillissement augmentent le rendement carcasse de 1 p. 100 (5859 p. $100, p<0,05)$ mais ne modifie pas le rendement de découpe $(72$ 74 p. 100). La castration induit une diminution de la quantité de viande totale produite par carcasse $(-13 \mathrm{p} .100, \mathrm{p}<0,01)$, cet effet ne touchant pas les morceaux de l'arrière-main $(11,2 \mathrm{~kg}$ à 24 mois, $12 \mathrm{~kg}$ à 30 mois). La composition de la viande n'est pas modifiée par la castration ou l'âge d'abattage ( 23 p. 100 de protéines, 0,7 p. 100 de lipides, 0,5 p. 100 de collagène). A 24 mois, la venaison de cerf rusa est plus tendre $(+24$ p. $100, \mathrm{p}<0,01)$ et moins foncée $(-21$ p. 100 , $p<0,01)$ qu'à 30 mois. Si les éleveurs de cerfs rusa castrent leurs jeunes mâles vers 1 an, il semble plus intéressant de les abattre à 24 mois.

Mots clés : Cervidae - Cervus timorensis russa - Cerf rusa - Castration Abattage d'animaux - Carcasse - Croissance - Viande de gibier - Nouvelle Calédonie.

\section{INTRODUCTION}

La présence du cerf rusa en Nouvelle-Calédonie remonte à 1870 , suite à l'introduction accidentelle de 12 animaux. S'inspirant du modèle néo-zélandais, la transition de la chasse à l'élevage démarra en 1987 avec l'appui de l'Agence de Développement rural et d'Aménagement foncier (ADRAF). Actuellement, la filière cerf s'articule autour d'une association d'éleveurs, l'Etablissement de l'Elevage de Cervidés de Nouvelle-Calédonie (EDEC), regroupant 12000 animaux répartis dans une vingtaine de fermes. L'Office de Commercialisation et d'Entreposage frigorifique (OCEF), organisme territorial, se charge

1. CIRAD-EMVT, BP 186 Nouméa, Nouvelle-Calédonie.

2. CIRAD-EMVT, 10, rue Pierre Curie, 94704 Maisons-Alfort Cedex, France.

* Adresse actuelle : CIRAD-EMVT/ERPA \& IGF/EBCD, c/o FTCC, Richmond Tower, 75/20 Sukhumvit Soi 26, Bangkok 10110, Thaillande.

Reçu le 14.3.1994, accepté le 21.3.1995. de l'abattage des animaux (abattoir agréé CEE de Bourail) et de la commercialisation de la venaison.

Limitée par un marché intérieur étroit et saturé par des circuits parallèles (chasse et braconnage), la filière s'est tournée naturellement vers l'exportation d'animaux vivants (Malaisie, Thaïlande) et de venaisons (CEE, Japon, Australie). Si l'exportation de reproducteurs vers la Malaisie démarra rapidement en raison d'une forte demande, l'exportation de venaison s'est heurtée à un marché largement occupé par les néo-zélandais qui, avec 900000 biches élaphes en production, offrent un produit disponible et de qualité standard.

Pour conquérir une part de marché, la filière cerf en Nouvelle-Calédonie doit offrir aux acheteurs potentiels un produit standard, de qualité hygiénique irréprochable. En raison de l'importance de la durée du rut chez cette espèce, les premiers travaux ont porté sur l'intérêt de castrer les jeunes mâles (daguets), l'abattage pendant le rut (juillet à novembre) présentant certains inconvénients : carcasses à odeur prononcée, manipulations d'animaux difficiles. Le but de cette étude est d'apprécier l'impact d'une castration pratiquée à un an sur la croissance et les performances d'abattage du jeune cerf rusa.

\section{MATÉRIEL ET MÉTHODES}

L'élevage expérimental de cerf rusa du CIRAD-EMVT se situe dans la Station zootechnique de Port-Laguerre, à une trentaine de $\mathrm{km}$ au nord de Nouméa, sur la façade ouest de la Grande Terre. La station bénéficie d'un climat de type tropical océanique, caractérisé par l'alternance irrégulière de trois saisons : chaude et humide (de janvier à avril), fraîche (de mai à août), puis sèche (de septembre à décembre). La pluviométrie et la température moyennes se situent respectivement aux alentours de $1200 \mathrm{~mm}$ et de $22^{\circ} \mathrm{C}$. L'année 1991 , période expérimentale, fut caractérisée par une pluviométrie déficitaire $(836 \mathrm{~mm})$ et une saison sèche marquée de 3 mois (octobre 1991 à mi-janvier 1992). En 1992, le niveau des précipitations se rapprocha des valeurs moyennes (1 $160 \mathrm{~mm}$ d'eau).

Le cadre de l'expérimentation est une zone améliorée de 10 ha, peu accidentée et non embuissonnée, avec un couvert graminéen à base de Brachiaria decumbens, Panicum maximum, Paspalum plicatulum, Setaria sphacelata où l'on trouve également en faible proportion des 
S. Le Bel P. Chardonnet D. Dulieu M. Salas

légumineuses spontanées (Desmodium canum, Desmanthus virgatus, Mimosa pudica) et introduites (Macroptilium atropurpureum, Neonotonia wigthii, Stylosanthes scabra).

\section{Les animaux}

Quarante-quatre faons mâles issus d'un même élevage sont introduits à la station à l'âge de 12 mois. Ils pèsent alors $24,6 \pm 3,7 \mathrm{~kg}$ (écarts extrêmes 19,0 à $32,5 \mathrm{~kg}$ ). Vingt des 44 daguets sont castrés par voie chirurgicale. Au cours des 18 mois d'expérimentation, le nombre de daguets castrés est passé de 20 à 17, un animal ayant eu un accident au cours d'une des premières pesées et 2 animaux s'étant temporairement échappés. Parmi les daguets entiers, 5 furent retenus comme futurs reproducteurs, done non abattus.

\section{Durée d'embouche}

La durée d'embouche de cet essai est conditionnée par le rut qui s'étend, en Nouvelle-Calédonie, de juillet à novembre. Le premier abattage eut lieu au bout de 52 semaines d'embouche avant le rut (en avril), les daguets étant âgés d'environ 24 mois. Le deuxième abattage eut lieu après 78 semaines d'embouche pendant le rut (en octobre), à l'âge de 30 mois environ.

Quatre groupes sont distingués (tabl. I) : 2 lots d'abattages Ab1 et Ab2 (abattage d'avril et d'octobre) et deux phases de croissance $\mathrm{Cr} 1$ et $\mathrm{Cr} 2$ : une première phase de croissance d'avril 1991 à avril 1992 pour tous les individus et une seconde phase de croissance d'avril 1992 à octobre 1992 pour le groupe Ab2.

\section{Conduite des animaux}

Les parcelles réservées aux daguets sont pâturées par rotation en adoptant la stratégie suivante:

- exploitation des graminées au stade montaison ;

- séjour des animaux de 10 à 15 jours sur une même parcelle ;

- gyrobroyage des refus ;
- fertilisation azotée à raison de $200 \mathrm{~kg} / \mathrm{ha} / \mathrm{an}$ d'urée en 4 fois ;

- repousse de 4 semaines en saison chaude et de 8 semaines en saison fraîche ;

- pierre à lécher dans chaque parcelle (ciment, sel et complément minéral vitaminé) ;

- pesée des animaux à l'entrée et à la sortie des parcelles.

En saison sèche, les daguets sont immobilisés sur une des parcelles où de l'ensilage de maïs $(0,73 \mathrm{UF} / \mathrm{kg}, 71 \mathrm{~g}$ $M A D / \mathrm{kg}$ ) leur est distribué pendant 8 semaines en novembre-décembre 1991 à raison de $0,7 \mathrm{~kg}$ MS/cerf/jour.

\section{Abattage et découpe des carcasses}

Un premier lot de daguets (moitié castrés et moitié entiers) est abattu en avril 1992 et un deuxième lot (moitié castrés et moitié entiers) en octobre 1992 pendant le rut. Acheminés la veille à l'abattoir, les daguets sont abattus en procédant à leur étourdissement au matador puis à la saignée, suivie d'une électro-stimulation de la carcasse pendant une minute. La carcasse est ensuite reprise par la chaîne de travail classique, avant d'être inspectée par les services vétérinaires et pesée à chaud. Le ressuyage s'effectue en chambre froide à $4^{\circ} \mathrm{C}$ pendant $72 \mathrm{~h}$. Les carcasses sont travaillées en salle de découpe $\left(15^{\circ} \mathrm{C}\right)$ selon la méthode suivante :

- décollement des épaules puis désossage ;

- section de la carcasse au niveau de la jonction sacro-lombaire, puis découpe des 2 cuissots qui sont désossés ;

- décollement des 2 filets-mignons en face ventrale et des 2 longes en face dorsale;

- le reste de la carcasse est décharnée pour fournir du ragoût.

Chaque pièce de découpe et le ragoût (2 épaules désossées, 2 cuissots désossés, 2 filets-mignons, 2 longes) sont emballés sous vide en sac rétractable CRYOVAC $\mathrm{BB} 4 \mathrm{~L}$, imperméable à l'oxygène et au $\mathrm{CO}_{2}$. Les mor-

TABLEAU I

Phases de croissance et périodes d'abattage

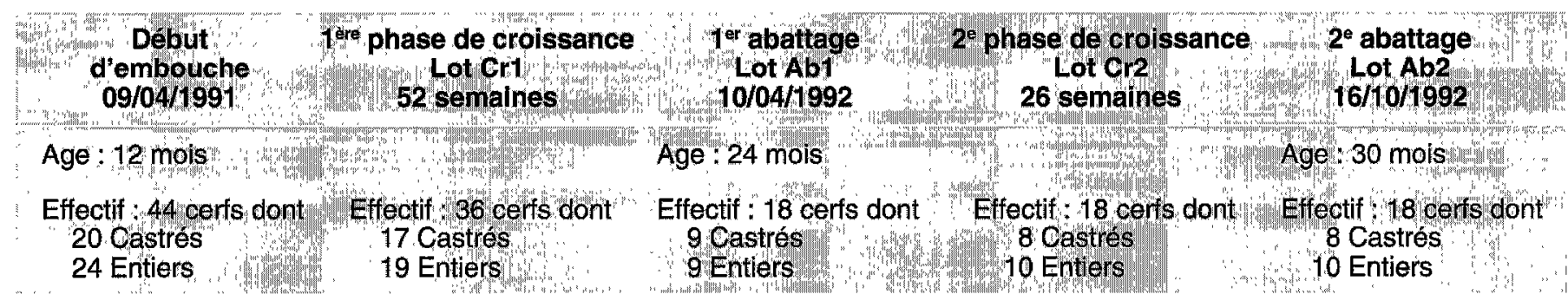


ceaux de découpe, identifiés (numéro de la carcasse) et pesés, sont stockés en chambre froide à $-18^{\circ} \mathrm{C}$ avant envoi en métropole.

\section{Analyses biologiques des pièces de découpe}

A chaque abattage, un échantillonnage de longes (une par daguet) maintenu à $0^{\circ} \mathrm{C}$ est acheminé au Centre technique de la Salaison, de la Charcuterie et des Conserves de Viandes de Maisons-Alfort (France) pour y être analysé.

\section{Mesure unitaire par échantillon :}

- de la teneur en humidité exprimée en p. 100 de matière fraîche (détermination de l'humidité par dessiccation à $103^{\circ} \mathrm{C}$ (NFV 04-401, janvier 1968) ;

- de la teneur en lipides exprimée en p. 100 de matière fraîche (détermination de la teneur en matière grasse totale par traitement à l'acide chlorhydrique et extraction à l'éther diéthylique (NFV 18-104, juin 1980) ;

- de la teneur en protides exprimée en p. 100 de matière fraîche (détermination de l'azote total selon la méthode de Kjeldahl, janvier 1968) ;

- de la teneur en collagène exprimée en p. 100 de matière fraîche (détermination de la teneur en L-hydroxyproline (NFV 04-405, avril 87)).

- de la teneur en pigments totaux exprimée en ppm de matière fraîche (méthode de Kaniecko) ;

- du $\mathrm{pH}$ par mesure de différences de potentiel (NFV 04408, janvier 1968).

Série de 12 mesures par échantillon de :

- la texture exprimée en centième de joules, mesurée par résistance du produit en utilisant I'INSTRON 6022 avec un outil en forme d'étoile à 5 branches tranchantes (méthode CTSCCV).

\section{Analyses statistiques}

Selon le type de paramètre étudié, on procède à deux types d'analyse statistique:

- mesure de l'effet du facteur "castration" sur la croissance, en recherchant l'existence de différences significatives par comparaison des moyennes de gain moyen quotidien (GMQ) entre les deux lots $\mathrm{Cr} 1$ et $\mathrm{Cr} 2$ selon le test $t$ (loi de Student-Fisher) appliqué aux petits échantillons ;

- mesure de l'effet des facteurs "castration" et "âge d'abattage" sur les performances d'abattage et la qualité biologique de la viande par analyse de variance (Anova, logiciel SPSS/PC+ V4.0, 1990).

\section{RESULTATS}

\section{Croissance et castration}

Les performances de croissance des deux catégories de cerfs, exprimées en (GMQ) figurent dans le tableau II.

\section{Première phase de croissance d'avril 1991 à avril 1992}

De 12 à 24 mois, le GMQ des daguets est de $82 \mathrm{~g} / \mathrm{j}$ chez les sujets castrés et de $91 \mathrm{~g} / \mathrm{j}$ chez les entiers. La faible différence de croît de $9 \mathrm{~g} / \mathrm{j}$ est significative sur cette période d'embouche de 12 mois $(t=3,35, p<0,01)$.

L'examen de la première partie du tableau II montre que cette première phase de croissance se découpe en deux temps. Jusqu'à la fin de la saison fraîche (périodes 1 et 2), la croissance des daguets castrés ou entiers est soutenue avec un GMQ supérieur à $100 \mathrm{~g} / \mathrm{j}$. En saison sèche (période 3), il diminue rapidement pour atteindre $51 \mathrm{~g} / \mathrm{j}$ pour les sujets castrés et $48 \mathrm{~g} / \mathrm{j}$ pour les entiers. Le retour de la saison des pluies (période 4) entraîne une faible reprise de la croissance, phénomène plus marqué chez les entiers (GMQ de $56 \mathrm{~g} / \mathrm{j}$ pour les castrés et de $72 \mathrm{~g} / \mathrm{j}$ pour les entiers). Les différences observées pendant ces 4 périodes ne sont pas significatives, sauf en saison fraîche (période 2).

En comparant le poids vif des jeunes mâles, il s'avère que la castration entraîne un effet négatif significatif $(\mathrm{t}=$ $2,63, p<0,01)$ de faible amplitude. Après 12 mois d'embouche, l'écart de poids vif entre les deux lots est de $3,2 \mathrm{~kg}$ en moyenne $(57,7 \mathrm{~kg} / 54,5 \mathrm{~kg})$, soit 5,5 à 5,9 p. 100 du poids vif du jeune mâle entier ou castré.

\section{Deuxième phase de croissance d'avril 1992 à octobre 1992}

De 24 à 30 mois, la croissance est ralentie. Le GMQ des daguets passe de 82 à $45 \mathrm{~g} / \mathrm{j}$ chez les individus castrés et de 91 à $63 \mathrm{~g} / \mathrm{j}$ chez les entiers. La différence de croît de $18 \mathrm{~g} / \mathrm{j}$ pendant cette période entre les deux catégories de daguets est significative $(t=2,52, p<0,05)$.

La lecture de la seconde partie du tableau II montre que cette phase de décélération de la croissance est marquée en saison fraîche (période 6) avec des GMQ inférieurs à $50 \mathrm{~g} / \mathrm{j}(33 \mathrm{~g} / \mathrm{j}$ pour les sujets castrés et $44 \mathrm{~g} / \mathrm{j}$ pour les entiers). L'écart de $3,2 \mathrm{~kg}$ entre les poids vifs des deux catégories d'animaux à 24 mois s'est accentué pour atteindre $6 \mathrm{~kg}(\mathrm{t}=3,17, \mathrm{p}<0,05)(68,5 \mathrm{~kg} / 62,5 \mathrm{~kg})$ au deuxième abattage à 30 mois, soit 8,8 à 9,6 p. $100 \mathrm{du}$ poids vif du jeune mâle entier ou castré.

De 12 à 30 mois, la vitesse de croissance moyenne est de $70 \mathrm{~g} / \mathrm{j}$ chez les daguets castrés et de $82 \mathrm{~g} / \mathrm{j}$ chez les entiers. La différence observée est significative $(t=3,17$, $p<0,01)$. 
TaBleau IV

Résultats des analyses de variances sur les principaux paramètres biologiques de la viande de cerfs rusa des lots Ab1 et Ab2 (avec l'âge et la castration comme facteurs)

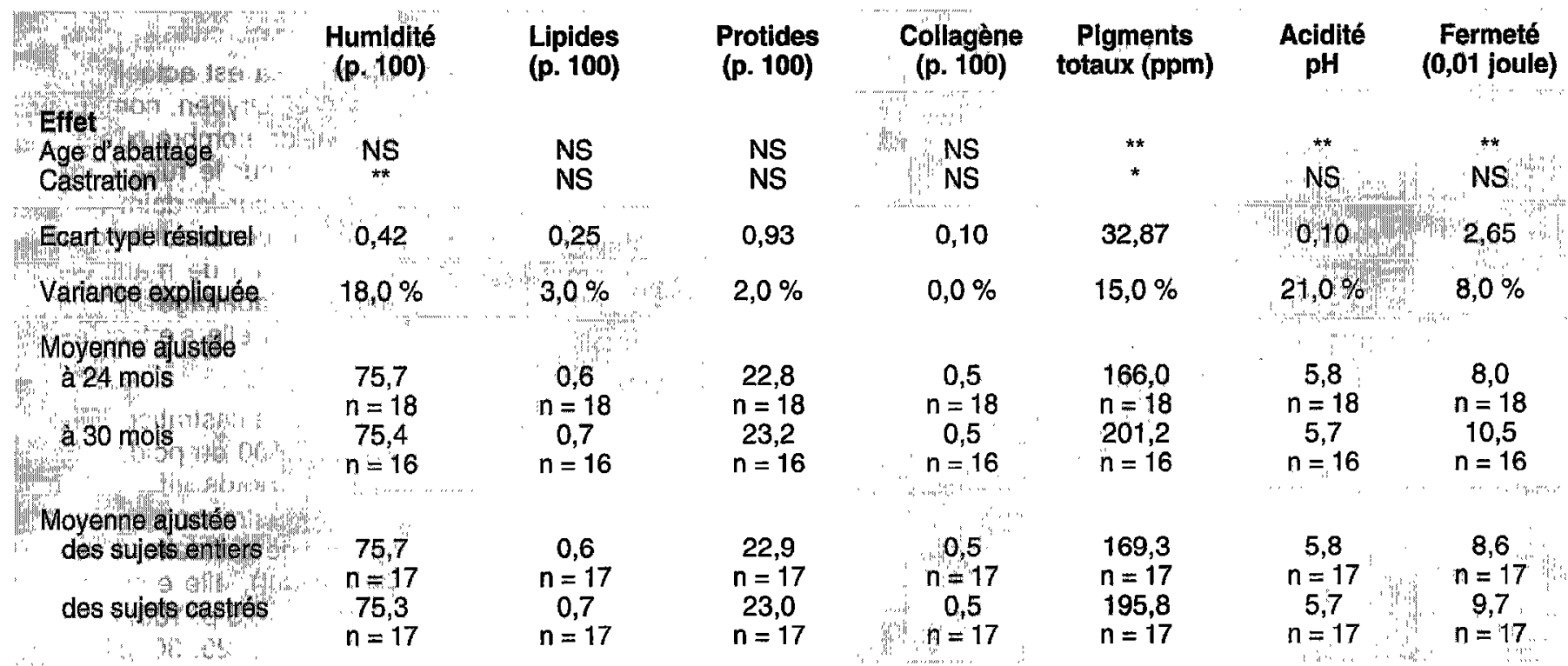

${ }^{*} p<0,05 ;{ }^{* *} p<0,01 ; N S p>0,05$ non significatif.

tive $(F=4,3, p<0,05)$ du rendement carcasse de 1 p. 100 (moyennes ajustées $58 / 59$ p. 100).

\section{Résultats de découpe}

Les résultats de découpe sont exposés dans le tableau III. Les facteurs âge d'abattage et castration n'ont pas d'effet significatif sur les résultats du rendement de découpe ( $F=0,46$ et $1,84, p>0.05)$. Quels que soient la date d'abattage ou l'état de l'animal (entier ou castré), le rendement de découpe se situe dans une fourchette de 72 à 74 p. 100. Avec une variance expliquée (R2) de 1 p. 100 , les variations enregistrées dépendent fortement des facteurs individuels.

Le choix d'abattre à 30 mois plutôt qu'à 24 s'accompagne d'une augmentation significative de la viande produite par carcasse de $4,8 \mathrm{~kg}(+20$ p. 100) $(F=44,7, p<0,01)$ [moyennes ajustées $23,7 / 28,5 \mathrm{~kg}$ ]. La quantité de viande de second choix produite par carcasse augmente de 32 p. $100(4 \mathrm{~kg})(F=59,9, p<0,01)$ [moyennes ajustées $16,5 / 12,5 \mathrm{~kg}]$, soit une progression de 8 p.100 (53 à 58 p. 100) entre 24 et 30 mois. De 24 à 30 mois, la quantité de viande de 1er choix augmente significativement $(F$ $=7,6, p<0,01)$ de 7 p. $100(800 \mathrm{~g})$ [moyennes ajustées $11,2 / 12 \mathrm{~kg}$ ], rapportée à la production de viande totale, la proportion de viande de ter choix diminue de 47 à 42 p. 100.

La castration entraîne une perte significative $(F=8,6$, $p<0,01)$ de $2 \mathrm{~kg}$ de viande totale produite par carcasse $(-7,4$ p. 100) [moyennes ajustées $24,9 / 26,9 \mathrm{~kg}$ ] et de viande de second choix (-13 p. 100) ( $F=14,3, p<0,01)$ [moyennes ajustées $13,3 / 15,3 \mathrm{~kg}$ ). La proportion de ce

type de viande est plus faible chez les daguets castrés (53 p. 100) que chez les entiers (57 p. 100). La castration n'a pas d'effet significatif ( $F=0,24)$ sur la production de viande de premier choix, la valeur des moyennes ajustées chez les daguets entiers et castrés étant quasi similaire $(11,7 / 11,5 \mathrm{~kg})$. A poids carcasse égal, les castrés produisent plus de viande de 1er choix que les entiers $(46,2$ p. 100 chez les castrés et 43,5 p. 100 chez les entiers).

\section{Qualité biologique de la viande et castration}

Le tableau IV expose les résultats des principaux paramètres biologiques de la viande de cerf rusa.

\section{Composition biochimique, facteurs nutritionnels}

\section{Humidité}

Quels que soient le type d'animal abattu ou la saison d'abattage, la teneur en eau des carcasses est comprise entre 75,3 et 75,7 p. 100 . Le facteur âge d'abattage n'influe pas sur la teneur en eau de la viande $(F=3,7$, $p>0,05$ ) et la castration entraîne une diminution significative $(F=7,7, p<0,01)$ quoique minime $(0,5 p .100)$ de ce paramètre.

Teneur en lipides, protides et collagène

Les facteurs âge d'abattage et castration n'ont pas d'effet significatif sur les teneurs en lipides, protides ou collagène $(F=1,5, p>0,05)$. Avec une variance expliquée de 0 à 3 
p. 100 , les variations enregistrées dépendent fortement des facteurs individuels. Indépendamment de la castration ou de la saison d'abattage, la viande de daguet rusa apparaît comme une venaison riche en protides (23 p. 100), pauvre en lipides $(0,6-0,7$ p. 100) et en collagène $(0,5 \mathrm{p}, 100)$.

\section{Teneur en pigments totaux}

Le choix d'abattre à 30 mois plutôt qu'à 24 entraîne la production d'une viande significativement plus foncée $(F=9,7, p<0,01)$, la teneur en pigments totaux augmentant de 21 p. 100 (moyennes ajustées 166/201,2 ppm). La castration entraîne le même type de phénomène $(F=5,5, p<0,05)$, la teneur en pigments totaux étant de 16 p. 100 plus élevée chez les individus castrés (moyennes ajustées 169,3/195,8 ppm).

\section{Acidité}

L'âge d'abattage a un effet significatif ( $F=17,3, p<0,01$ ) sur l'acidité des carcasses alors que la castration serait sans effet $(F=0,9, p>0,05)$. Néanmoins les résultats des moyennes ajustées montrent que les variations restent faibles, la valeur du $\mathrm{pH}$ des carcasses se situe dans une fourchette de 5,7 à 5,8 .

\section{Mesures de texture pratiquées sur les longes}

L'abattage à 24 mois donne une viande significativement plus tendre $(F=7,9, p<0,01)$ qu'à 30 mois d'environ 24 p. 100 (moyennes ajustées 8/10,5 centième de joule). La castration n'entraîne aucune modification significative $(F=1,5, p>0.05)$.

\section{DISCUSSION}

\section{Croissance et castration}

Dans le présent essai, le GMQ observé est en moyenne inférieur à $100 \mathrm{~g} / \mathrm{j}$ quel que soit le lot, la castration ayant néanmoins un effet dépresseur. Un tel niveau de croissance correspond à un type d'élevage extensif. D'autres travaux menés en Nouvelle-Calédonie et en Australie ont montré qu'il était possible, dans un système de production plus intensif, d'enregistrer des performances plus élevées que celles observées ici. En Australie, Woodford $(36,37,38)$, sur kikuyu (Pennisetum clandestinum) irrigué avec apport de céréales, enregistre des performances de 230g/j de 0 à 6 mois et de $175 \mathrm{~g} / \mathrm{j}$ de 6 à 12 mois. Grimaud et Chardonnet (18) en Nouvelle-Calédonie rapportent des performances proches, $180 \mathrm{~g} / \mathrm{j}$ sur des daguets de 4 à 16 mois nourris avec une ration fourrage (75 p. 100) et concentré (25 p. 100). Les daguets dans ce cas affichent un poids vif de $40 \mathrm{~kg}$ à 6 mois, $70 \mathrm{~kg}$ à 12 mois et $92,5 \mathrm{~kg}$ à 16 mois.

D'autres études conduites en Malaisie (24) rapportent des performances proches de celles enregistrées dans le présent essai avec des poids vifs de $40,5 \mathrm{~kg}$ à 15 mois, les daguets étant nourris de 6 à 15 mois à base de granulés, patates douces et d'herbe à éléphant (Pennisetum purpureum).

L'impact de la castration du cerf rusa est actuellement à l'étude en Australie (20,21, 22, 32, Dryden, com. pers.). Elle est pratiquée néanmoins par de nombreux éleveurs avant la puberté, vers 10-12 mois sur le rusa (Dryden, Hart, com. pers., 1992) et 4-6 mois sur le daim $(11,12$, $27,28,30,31$ ) pour des raisons de commodités d'élevage (diminution de l'agressivité, facilité de manipulation des jeunes mâles en élevage et à l'abattage). Elle reste exceptionnelle avec le cerf rouge où elle s'effectue vers 6-7 mois (19).

Chez cette dernière espèce $(7,19)$, la castration entraîne une perte de poids atteignant 9 p. 100 du poids vif à 16 mois $(84 / 76 \mathrm{~kg})$ et $17 \mathrm{p} .100 \mathrm{du}$ poids vif à 27 mois $(118 / 98 \mathrm{~kg})$. Avec le daim, où la castration est couramment pratiquée pour des raisons de conduite d'élevage (possibilité d'abattage pendant le rut), elle entraîne une perte de poids vif de $3 \mathrm{~kg}$ à 17 mois $(6,8 \mathrm{p} .100)$ et de 6,5 $\mathrm{kg}$ à 24 mois $(10,2$ p. 100) $(26,27,28,29,30,31)$. Dans l'essai, la castration à l'âge d'un an induit le même type de phénomène, la perte de poids vif n'excédant pas $3 \mathrm{~kg}$ à 24 mois ( 5 p. 100 du poids vif) et s'accentuant par la suite pour atteindre à 30 mois 6 à $8 \mathrm{~kg}(8,8$ à 11,7 p. 100 du poids vif). Dans des conditions alimentaires permettant des croissances plus élevées, Sookhareea et al. (32) montrent qu'une castration pratiquée précocement ( 27 semaines) n'entrave pas la croissance de daguets rusa abattus à 19 mois $(81,7$ à $84,8 \mathrm{~kg})$.

\section{Performances et âge d'abattage}

Dans la présente étude, l'abattage à 30 mois entraîne une augmentation du poids de carcasse de $6,1 \mathrm{~kg}(38,8$ $\mathrm{kg}$ à 30 mois, $32,7 \mathrm{~kg}$ à 24 mois) et un gain de $4,8 \mathrm{~kg}$ de viande produite à la découpe $(28,5 \mathrm{~kg}$ à 30 mois, $23,7 \mathrm{~kg}$ à 24 mois). Le gain de viande réalisé concerne surtout les morceaux de second choix $(+32$ p. 100), contrairement à ceux de premier choix $(+7 p$. 100). Les carcasses des daguets abattus à 24 mois dans le présent essai sont plus légères que celles produites, à âge égal, par les éleveurs australiens $(36,37,38$, Hart, com. pers., 1992) avec un poids moyen de 45 à $48 \mathrm{~kg}$. Cette différence s'explique par le régime alimentaire basé sur l'exploitation du pâturage et le choix du maigre disponible pour ce type d'embouche en avril 1991. Le pic de naissance du rusa en Nouvelle-Calédonie se situant de mi-avril à mijuin (2), les jeunes daguets âgés de 10 à 12 mois affichent en début d'essai un poids vif inférieur à ceux enregistrés dans les élevages du Queensland $(36,37,38)$. Cette situation reflète celle de l'élevage de cerfs calédoniens où le faon sevré est sous-alimenté pendant les 3 à 5 mois de la saison sèche (23). Une alternative pour 
réduire la durée d'embouche serait de maintenir la croissance des faons pendant cette période en les supplémentant.

Dans la pratique, les éleveurs de cerfs optent pour un abattage précoce de leurs animaux vers 2 ans. Le cerf rusa est abattu à 24 mois en Australie (Hart, com. pers, $1992 ; 36,37,38$ ) ou à l'île Maurice (3), le daim à 17 mois $(12,26,27,28,29,30,31)$, le chital entre 13 et 18 mois (11) et le cerf rouge entre 14-15 mois ou 26-27 mois selon son état de développement $(1,7,8,9)$. Certains travaux chez le cerf rusa $(18,36,37,38)$ ont montré néanmoins qu'il était possible de raccourcir la durée d'embouche en abattant les daguets vers 14 mois (carcasse de $45 \mathrm{~kg}$ ).

En ce qui concerne la carcasse, on cite des rendements de 60 à 62 p. 100 chez des daguets rusa abattus à 13-15 mois et de 61 p. 100 à 24 mois $(36,37,38)$. Ces résultats sont supérieurs à ceux enregistrés ici où le rendement est de 58 p. 100 à 24 mois et de 59 p. 100 à 30 mois. Cette différence peut s'expliquer en raison du procédé d'abattage des cerfs en Australie et Nouvelle-Zélande (21) où la carcasse est pesée avec la fressure. Mulley et English (31) distinguent deux types de rendement chez le daim abattu à 17 mois, un rendement d'abattage de 60 à 61 p. 100 calculé en rapportant le poids de la carcasse plus fressure au poids vif de l'animal et un rendement carcasse de 55 à 56 p. 100 où le calcul est basé sur un poids de la carcasse débarrassée de la fressure.

\section{Performances d'abattage et castration}

Dans le présent essai, la castration entraîne une diminution du poids de la carcasse de 6 p. 100. La perte de viande induite $(-7,4$ p. 100) affecte l'avant-main et le ragoût $(-13$ p. 100) et non l'arrière-main.

Chez les autres espèces de Cervidés, la castration des daguets entraîne une diminution du poids de la carcasse pouvant atteindre 17 p. $100 \mathrm{chez}$ le cerf rouge de 27 mois $(7,19)$ et 7 à 8 p. 100 chez le daim de 17 mois $(11$, 12,31 ). De récents travaux (32) confirment l'effet dépresseur de la castration sur le rendement de la carcasse mais soulignent que si les animaux sont abattus précocement vers 19 mois, on n'observe pas de diminution du poids de la carcasse à chaud ou à froid. Pour le daim comme pour le cerf rusa dans cet essai, la diminution du poids de la carcasse concerne des morceaux de l'avantmain, les morceaux de découpe de l'arrière-main n'étant pas affectés par la castration (Woodford, com. pers. in GRIMAUD (15) ; 30).

\section{Venaison et castration}

En comparant les valeurs obtenues avec celles des autres espèces bouchères (33), on constate que la longe de cerf rusa, avec 1 p. 100 de gras intramusculaire, plus de 22 p. 100 de protides et moins de 0,5 p. 100 de colla- gène, s'apparente aux viandes dites "maigres" comme la viande blanche parée de dinde ou de poulet, ou le maigre sans gras et nerf visible de boeuf ou de cheval.

Cette description analytique est voisine de celle des autres espèces de Cervidés (9). La longe de rusa apparaît néanmoins plus maigre avec 0,7 p. 100 de lipides que la longe de daim (1,3 p. 100 de lipides) (25), la viande de chevreuil (selle : 3,6 p. 100 de lipides) ou la viande de cerf rouge avant rut (venaison : 3,3 p. 100 de lipides) (33). Des travaux récents (34) montrent qu'il est possible d'avoir des teneurs en lipides similaires chez le cerf rouge $(0,4$ p. 100 pour la longe) lorsque celui-ci est abattu après le rut. D'après English (11), les espèces tropicales de Cervidés comme le rusa ou le chital se distingueraient des autres espèces tempérées par le faible dépôt de gras au niveau de la carcasse, dépôt qui peut atteindre 8 à 12 p. 100 chez le daim et le cerf rouge.

La castration n'a pas entraîné d'augmentation de dépôt de gras intramusculaire, quelle que soit la période d'abattage. Chez les espèces de Cervidés tempérés, on constate l'effet contraire avec une augmentation du dépôt de gras au niveau de la carcasse de 16 p. 100 chez le cerf rouge (7) et de 2 à 10 p. 100 chez le daim (27).

Avec moins de 0,5 p. 100 de collagène, la longe de cerf rusa castré ou entier appartient à la catégorie des viandes tendres, pauvres en tissu conjonctif, dites à rôtir ou à griller (14). En comparaison, les viandes de boeuf ou de cheval contiennent 2 à 4,5 p. 100 de collagène $(6,10)$; seules, les viandes blanches parées de volailles affichent des teneurs inférieures à 1 p. 100.

Les mesures de texture effectuées dans cet essai montrent que la tendreté de la viande de cerf rusa est affectée par le vieillissement et non par le rut ou la castration. Sookhareea et al. (32) aboutissent aux mêmes résultats chez le jeune mâle rusa castré. Leurs travaux diffèrent néanmoins en ce qui concerne l'impact de la castration sur la couleur de la viande: dans leur cas, elle l'éclaircit alors que dans le présent essai, elle l'assombrit. Chez les espèces tempérées comme le cerf élaphe (34), le rut se traduit au contraire par une diminution significative de la tendreté des muscles long-dorsaux ou semi-membraneux.

\section{CONCLUSION}

La castration du daguet rusa vers $10-12$ mois entraîne une perte de poids vif d'environ $3 \mathrm{~kg}$ à 24 mois et de $6 \mathrm{~kg}$ à 30 mois. Chez les animaux abattus à 24 mois, la castration est compatible avec la fourniture de carcasses de bonne qualité bouchère : la perte en viande liée à cette pratique est limitée à $2 \mathrm{~kg}$ et ne concerne pas les meilleurs morceaux de venaison situés dans l'arrièremain. La castration n'affecte pas la qualité de la venaison du cerf rusa qui est une viande diététique riche en protéines, pauvre en lipides et collagène, destinée à un type de cuisson rapide (grillades). Chez les éleveurs de cerfs 


\section{S. Le Bel P. Chardonnet D. Dulieu M. Salas}

rusa pratiquant la castration des jeunes mâles, les résultats de cet essai recommandent donc d'abattre précocement les animaux vers 24 mois. Un abattage à ce stade de développement permet notamment de fournir une viande plus tendre et moins foncée qu'un abattage tardif à 30 mois.

\section{BIBLIOGRAPHIE}

1. BRELURUT A., PINGARD A., THÉRIEZ M., 1990. Le cerf et son élevage. Paris, INRA, Maisons-Alfort, France, Editions du Point Vétérinaire, 143 p

2. CHARDONNET P., 1988. Etude de faisabilité technique et économique de l'élevage de cerfs en Nouvelle-Calédonie. Maisons-Alfort, France, IEMVT/ADRAF, $282 \mathrm{p}$.

3. CHARDONNET P., 1988. Observations et réflexions sur l'élevage de cerfs à l' Ile Maurice. Maisons-Alfort, France, IEMVT, 36 p. (Rapport IEMVT)

4. CHARDONNET P., GRIMAUD P., AGATHE NERINE S., 1990. Commercial rusa deer production in New Caledonia. In: Proc. Second Inter. Wildlife Ranching Symposium Edmonton, Alberta, Canada, June 1990, p. 109-112.

5. CHARDONNET P., LARTIGES A., 1993. Les habitats et la faune terrestre vertćbrés de Nouvclle-Calédonic (1c̀ro partic), Bull. mens. Off. Natn. Chasse, $180: 41-45$

6. CTSCCV, 1983. Composition des matic̀res premic̀res, In : l'Encyclopédie de la char̃cuterie. Paris, France, Soussana, p. 150-152.

7. DREW K., GREER G., 1977. Venison production and carcass composition of red deer. N.Z. Agric. Sci., 11 p.

8. DREW K.R., FENNESSY P.F., 1986. Venison research - Carcass fcature proceeding and packaging. Deer Branch Course $n^{\circ} 3$. Roturua, Nouvelle Zélande, p.17-34.

9. DREW K.R., STEVENSON J.M., FENNESSY P.F., 1991. Venison. A marketable product. Deer Branch Course n ${ }^{\circ}$. Sydney, Australie, p. 31-35.

10. DUMONT B.L.,1986. La viande de bouf : structure et tendreté. Pour la Science, p. 88-96.

\section{ENGLISH A.W., 1990. The Chital challenge. N.Z. Deer Farmer, p. 53-64.}

12. ENGLISH A.W., 1990. Management strategies and health programs for farmed fallow deer in Australia. Deer branch course $n^{\circ}$, p. 116-127.

13. FLECHER T.J., 1986. In: Management and Diseases of Deer, Section 2: Reproduction. Veterinary Deer Society, p. 15-21.

14. FRAYSSE J.L., DARRÉ A., 1990. Produire des viandes. Volume 1 : Sur quelles bases économiques et biologiques ? Paris, France, Technique et documentation Lavoisier, 374 p. (Coll. agriculture d'aujourd'hui)

15. GRIMAUD P., 1991. Le cerf rusa, Cervus timorensis russa en Australie. Nouméa, Nouvelle-Calédonie, CIRAD, Mandat de gestion, 35 p. (Etudes et synthèses)

16. GRIMAUD P., 1992. Croissance et ingestion chez les bichettes et daguets. Les Journées Techniques de Port-Laguerre sur l'élevage du Rusa (2). Nouméa, Nouvelle-Calédonie, CIRAD, Mandat de gestion. (Etudes et synthèses)

17. GRIMAUD P., 1992. Ingestion comparée. Les Journées Techniques de Port-Laguerre sur l'élevage du Rusa (2). Nouméa, Nouvelle-Calédonie, CIRAD, Mandat de gestion. (Etudes et synthèses)
18. GRIMAUD P., CHARDONNET P., 1989. Comportement alimentaire du cerf rusa en croissance recevant une ration à base de graminée (Brachiaria mutica). Actes du XVIe congrès international des herbages, Nice, France, 4-11 octobre 1989. Versailles, France, Association française pour la production fourragères, INRA, p. 1281-1282.

19. HERNANDEZ J.C., 1984. L'élevage des Cervidés en Nouvelle-Zélande. Thèse $n^{\circ} 27$, Ecole nationale vétérinaire de Toulouse, Toulouse, France, $179 \mathrm{p}$.

20. LE BEL S., 1992. Exploitation de la biomasse fourragère, méthodes et résultats technico-économiques. Les Journées Techniques de Port-Laguerre sur l'élevage du Rusa (1). Nouméa, Nouvelle-Calédonie, CIRAD, Mandat de gestion. (Etudes et synthèses)

21. LE BEL S., 1992. Embouche de daguets à l"herbe, effet de la castration. Les Journées Techniques de Port-Laguerre sur l'élevage du Rusa (2). Nouméa, Nouvelle-Calédonie, CIRAD, Mandat de gestion. (Etudes et synthèses)

22. LE BEL S., 1993. Rapport de mission en Australie du 16 nov. au 5 déc. 1992. Nouméa, Nouvelle-Calédonie, CIRAD, Mandat de gestion, 27 p. (Etudes et synthèses)

23. LE BEL S., DULIEU D., 1993. Rearing rusa deer in New Caledonia, a first attempt at relating feed management to the system of production. In: World Deer Congress, Christchurch, New Zealand, February 1993. New Zealand deer Farmers Association, p. 143-148.

24. MOHD NASIS A., ARIFF OMAR M., 1991. Body measurements and weights of Cervus timorensis russa. Pahang DM, Malaisie, Livestock research Division, Mardi Bukit Ridan. (in press)

25. MOJTO J., CARTUSEK V., PALANSKA O., LAHUCKY R., 1992 Carcass and meat quality of fallow deer. In: proceeding 38th International Congress of Meat Science and Technology, Clermont-Ferrand, France, August 23-28, 1992, Vol. 2. Helsinski, Finlande, Congress Secretariat of ICOMST, p. 97-100.

26. MULLEY R.C., 1984. The reproductive performance of farmed fallow deer in New South Wales. Proceeding Number 72, Deer Refresher Course, The Post-graduate Committee in Veterinary Science, The University of Sydney, Australie, p. 461-480.

27. MULLEY R.C.,1988. Reproduction and performance of farmed fallow deer (Dama dama) Ph.D. Thesis, The University of Sydney, Australie.

28. MULLEY R.C., 1990. Castration. In: Principles of Practical Deer Far ming. Proc. Deer Breeders Assoc. of Western Australia, Perth, p. 74-86.

29. MULLEY R.C, ENGLISH A.W., 1985. The effects of castration of fallow deer (Dama dama) in New South Wales. Anim. Prod., 41: 359-361.

30. MULLEY R.C, ENGLISH A.W., 1990. Evaluation of carcass changes to fallow bucks castrated pré-puberally. In : Brown D. ed, Proc. Inter. Symp. Biology of deer, Mississipi State University; USA, June 1990, p. $238-243$.

31. MULLEY R.C., ENGLISH A.W., 1991. Fallow deer carcass production. Deer Branch Course n ${ }^{\circ} 8$. Sydney, Australie, p. 68-75.

32. SOOKHAREEA D.G.T., et al, 1993. A study of carcass characteristics and meat quality of castrate and entire javan rusa stags. CSIRO, $5 \mathrm{p}$. (The Australian Meat Industry Research Conference $\mathrm{II}^{\prime} 8$ )

33. SOUCI S.W., FACHMANN W., KRAUT H., 1986. La composition des aliments. Tableaux des valeurs nutritives 1986-1987. 3e edn revue et complété par SCHERTZ H., KOOS G., SENSER F., Stuggart, Allemagne, Wissenschaftliche Verlagsgesellschaft mbH, p. 230-344.

34. STEVENSON J.M., SEMAN D.L., LITTLEJOHN R.P., 1992. Seasonal variation in venison quality of mature farmed red deer stags in New Zealand. J. Anim. Sci., 70: 1389-1396. 
35. THORLEY $\Lambda ., 1990$. The moluccan rusa dilemma. Deer Farmer, March 1990: 31-34.

36. WOODFORD K., 1991. Dry argument supports Australian Rusa Farming. Deer Farmer, 79: 37-39.

LE BEL (S.), CHARDONNET (P.), DULIEU (D.), SAlAS (M.). Impact of castration of rusa stags on growth rate, slaughtering and meat quality at 24 and 30 months. Revue Elev. Méd. vét. Pays trop., 1995, 48 (1): $85-93$

In young male rusa stags (Cervus timorensis russa) reared for slaughter, breeders consider rutting a constraint. The aim of the trial was to study the zootechnical impact of castration. Seventeen castrated (C) and nineteen entire (E) 12-month-old rusa Javanese stags were pasture-reared and slaughtered at 24 or 30 months of age. Castration reduces live weight at 24 months (C: $54.5 \pm 1.9 \mathrm{~kg}, \mathrm{E}: 58.5 \pm 2.3 \mathrm{~kg}$, $p<0.01)$ and 30 months $(C: 62.5 \pm 3.0 \mathrm{~kg}, \mathrm{E}: 68.5 \pm 2.3 \mathrm{~kg}, \mathrm{p}<0.01)$. From 12 to 24 months, the growth rate reaches $82 \pm 3$ g per day (C) and $91 \pm 1 \mathrm{~g}$ per day (E). From 24 to 30 months, the growth rate is reduced to $45 \pm 6 \mathrm{~g}$ per day (C) and $63 \pm 12 \mathrm{~g}$ per day (E). Castration and age increase the carcass yield by $1 \%(58-59 \%, p<0.05)$ but not the cutting yield (72-74 \%). Castration reduces the total meat produced per carcass by $13 \%(p<0.01)$ but this does not affect the cuts of the hindquarter $(11.2 \mathrm{~kg}$ at 24 months, $12 \mathrm{~kg}$ at 30 months). Meat composition is not affected by castration and age of slaughtering. The venison of entire or castrated rusa deer is a protein-rich $(23 \%)$, low-fat $(0.7 \%)$ and low-collagen $(0.5 \%)$ meat. The venison is more tender $(+24 \%, \mathrm{p}<0.01)$ and lighter $(-21 \%, \mathrm{p}<0.01)$ at 24 months than at 30 months. If rusa stag breeders castrate young stags at one year old, the best time to slaughter them seems to be at 24 months.

Key words : Cervidae - Cervus timorensis russa - Rusa deer - Castration - Slaughtering - Carcass - Growth - Game meet - New Caledonia.
37. WOODFORD K., 1991. Deer production in Tropical and Subtropical environments. Deer branch of the NZ veterinary association. Deer Branch Course $\mathrm{n}^{\circ} 8$. Sydney, Australie, p. 85-93.

38. WOODFORD K., DUNNING A., 1990. Production cycles and characteristics of rusa deer in Queensland, Australia, In : Brown D. ed., Proc. Inter. Symp. Biology of deer, Mississipi State University, USA, June 1990 , p. $197-202$.

LE BEL (S.), CHARDONNET (P.), DULIEU (D.), SALAS (M.). Impacto de la castración del ciervo rusa sobre las caracteristicas del crecimiento y de la matanza y sobre la calidad biologica de la carne, a los 24 y 30 meses. Revue Élev. Méd. vét. Pays trop., 1995, 48 (1) : 85-93

En el joven ciervo rusa macho (Cervus timorensis russa) destinado a matadero, el celo es considerado por el criador como un obstáculo. El objetivo de este experimento, es el de apreciar el impacto zootécnico de la castración. Diecisiete ciervos rusa de Java, castrados (C) y diecinueve enteros $(E)$, de 12 meses de edad, fueron alimentados con pasto y sacrificados a los 24 meses o a los 30 meses. La castración indujo una disminución del peso vivo a 24 meses (C:54,5 $\pm 1,9 \mathrm{~kg}$, $\mathrm{E}: 58,5 \pm 2,3 \mathrm{~kg}, \mathrm{p}<0,01)$ y a 30 meses $(\mathrm{C}: 62,5 \pm 3,0 \mathrm{~kg} \mathrm{E}: 68,5 \pm$ $2,3 \mathrm{~kg}, \mathrm{p}<0,01)$. De los 12 a los 24 meses, el crecimiento es de $82 \pm$ $3 \mathrm{~g} / \mathrm{d}$ (C) y de $91 \pm 1 \mathrm{~g} / \mathrm{d}$ (E). De los 24 a los 30 meses, éste disminuye para no alcanzar más que $45 \pm 6 \mathrm{~g} / \mathrm{d}$ (C) y $63 \pm 12 \mathrm{~g} / \mathrm{d}$ (E). La castración y el envejecimiento aumentan el rendimiento de la carcaza de 1 p. $100(58-59$ p. 100, p $<0,05)$, pero no modifica el rendimiento del corte (72-74 p. 100). La castración provoca una disminución de la cantidad de carne total producida por carcaza $(-13$ p. 100, p < 0,01$)$, sin afectar las porciones posteriores $(11,2 \mathrm{~kg}$ a 24 meses, $12 \mathrm{~kg}$ a 30 meses). La composición de la carne no es modificada por la castración, ni por la edad de la matanza ( 23 p. 100 proteínas, 0,7 p. 100 lípidos, 0,5 p. 100 colágeno). A los 24 meses la carne de caza mayor del ciervo rusa es más suave $(+24 \mathrm{p} .100, \mathrm{p}<0,01)$ y menos oscura (- 21 p. 100, p < 0,01) que a los 30 meses. Si los criadores de ciervos rusa castran sus machos jóvenes hacia la edad de un año, parece más interesante sacrificarlos a los 24 meses.

Palabras clave : Cervidae - Cervus timorensis russa - Venado rusa Castración - Sacrificio - Canal animal - Crecimiento - Carne de animales de caza - Nueva Caledonie. 